\title{
ON COMMUTATIVE HOMOGENEOUS VECTOR BUNDLES ATTACHED TO NILMANIFOLDS
}

\author{
ROCÍO DÍAZ MARTÍN AND LINDA SAAL
}

\begin{abstract}
The notion of Gelfand pair $(G, K)$ can be generalized by considering homogeneous vector bundles over $G / K$ instead of the homogeneous space $G / K$ and matrix-valued functions instead of scalar-valued functions. This gives the definition of commutative homogeneous vector bundles. Being a Gelfand pair is a necessary condition for being a commutative homogeneous vector bundle. In the case when $G / K$ is a nilmanifold having square-integrable representations, a big family of commutative homogeneous vector bundles was determined in [Transform. Groups 24 (2019), no. 3, 887-911]. In this paper we complete that classification.
\end{abstract}

\section{INTRODUCTION}

A homogeneous space $G / K$ is called commutative, and the pair $(G, K)$ is called a Gelfand pair, when $G$ is a locally compact group, $K$ is a compact subgroup of $G$, and the convolution algebra $L_{0}^{1}(G)$ is commutative. Here $L_{0}^{1}(G)$ denotes the Banach algebra of $L^{1}$ functions on $G$ satisfying $f\left(k x k^{\prime}\right)=f(x)$ for $x \in G$ and $k, k^{\prime} \in K$, where the product is the usual convolution $(f * h)(g)=\int_{G} f(x) h\left(x^{-1} g\right) d x$ on $G$ (where $d x$ denotes the Haar measure on $G$ ).

By a nilmanifold we mean a differentiable manifold on which a nilpotent Lie group acts transitively. By a commutative nilmanifold we mean a commutative space $G / K$ such that $G$ is a Lie group and a closed nilpotent subgroup $N$ of $G$ acts transitively (see e.g. 16 ). In that notation, if $G / K$ is simply connected then $N$ acts simply transitively on $G / K$ and $G$ is the semidirect product $K \ltimes N$ (see [15. Theorem 13.1.6]). It is shown in [1 that if $(K \ltimes N, K)$ is a Gelfand pair then $N$ must be abelian or two-step nilpotent.

Those definitions are associated to the commutative property of the algebra $L_{0}^{1}(G)$ of scalar-valued functions. For the vector-valued case we have analogous definitions.

Again, let $G$ be a Lie group and $K$ a compact subgroup of $G$. It is well known that all the homogeneous vector bundles over the homogeneous space $G / K$ are

2020 Mathematics Subject Classification. 22E25, 22F30, 43A85, 47 A67.

The research for this article has mainly been carried out while R. Díaz Martín was holding a fellowship from CONICET. 
described by taking finite-dimensional representations $\left(\tau, W_{\tau}\right)$ of $K$ (see [14, Section $5.2])$. Indeed, if we consider the equivalence relation over $G \times W_{\tau}$ given by $(g k, w) \sim$ $(g, \tau(k) w)$ for $g \in G, w \in W_{\tau}$, and $k \in K$, the space of equivalence classes $E_{\tau}$ has an structure of homogeneous vector bundle over $G / K$. Moreover, each homogeneous vector bundle over $G / K$ is isomorphic to $E_{\tau}$ for some representation $\left(\tau, W_{\tau}\right)$ of $K$ of finite dimension.

The space of compactly supported smooth sections of the homogeneous vector bundle $E_{\tau}$ is naturally identified with the space of functions $u$ on $\mathcal{D}\left(G, W_{\tau}\right)$ such that $u(x k)=\tau(k)^{-1} u(x)$ for $k \in K$ and $x \in G$. It follows from the Schwartz kernel theorem that every linear operator $T$, continuous with respect to the standard topologies, mapping $\mathcal{D}$-sections of $E_{\tau}$ into $\mathcal{D}^{\prime}$-sections of $E_{\tau}$ and commuting with the action of $G$ on $E_{\tau}$, can be represented in a unique way as a convolution operator

$$
(T(u))(g)=u * F(g):=\int_{G} F\left(x^{-1} g\right) u(x) d x \quad \forall g \in G,
$$

where $F$ is a distribution, $F \in \mathcal{D}^{\prime}\left(G\right.$, $\left.\operatorname{End}\left(W_{\tau}\right)\right)$, satisfying

$$
F\left(k_{1} x k_{2}\right)=\tau\left(k_{2}\right)^{-1} F(x) \tau\left(k_{1}\right)^{-1} \quad \forall k_{1}, k_{2} \in K, x \in G .
$$

In particular, operators $T=T_{F}$ as in 1.1 with $F$ a function in $L^{1}\left(G, \operatorname{End}\left(W_{\tau}\right)\right)$ satisfying 1.2 can be composed with each other and

$$
T_{F_{2}} \circ T_{F_{1}}=T_{F_{1} * F_{2}} .
$$

We call $L_{\tau, \tau}^{1}(G$, End $(W \tau))$ the convolution algebra of integrable matrix-valued functions with property $(1.2)$.

Let $\left(\tau, W_{\tau}\right)$ be an irreducible unitary representation of $K$. Let $\widehat{K}$ denote the set of equivalence classes of irreducible unitary representations of the group $K$. The homogeneous vector bundle $E_{\tau}$ is called commutative, and the triple $(G, K, \tau)$ is also called commutative, when the algebra $L_{\tau, \tau}^{1}\left(G, \operatorname{End}\left(W_{\tau}\right)\right)$ is commutative. In particular, $(G, K)$ is a Gelfand pair when $(G, K, \tau)$ is a commutative triple with $\tau$ the trivial representation of $K$. Here $L_{\tau, \tau}^{1}\left(G, \operatorname{End}\left(W_{\tau}\right)\right)=L_{0}^{1}(G)$. It is shown in [1] that if $G / K$ is connected and if there exists $\tau \in \widehat{K}$ such that $(G, K, \tau)$ is a commutative triple then $(G, K)$ is a Gelfand pair. Therefore, in most cases, being a Gelfand pair is a necessary condition to give rise to commutative triples. When $\tau$ is a character of $K$ and the triple $(G, K, \tau)$ is commutative, these cases are also known as twisted Gelfand pairs with respect to the character $\tau$. Finally, we say that $(G, K)$ is a strong Gelfand pair if $(G, K, \tau)$ is commutative for every $\tau \in \widehat{K}$.

In the case where $L_{\tau, \tau}^{1}\left(G, \operatorname{End}\left(W_{\tau}\right)\right)$ is a commutative algebra, the spherical analysis consists of the computation of the continuous characters of such convolution algebra and this gives rise to a kind of simultaneous "diagonalization" of all the operators $T_{F}$ (see e.g. [2, 7]).

From now on we concentrate on homogeneous vector bundles associated to nilmanifolds.

The classification of Gelfand pairs $(K \ltimes N, K)$ with $N$ nilpotent was completed by E. Vinberg. In the notable article [13] are exhibited all the Gelfand pairs $(K \ltimes N, K)$ that are irreducible and maximal. On the one hand, the irreducibility means that 
the center $\mathfrak{z}$ of the Lie algebra $\mathfrak{n}$ of $N$ must be $\mathfrak{z}=[\mathfrak{n}, \mathfrak{n}]$ and $K$ acts irreducibly on $\mathfrak{n} / \mathfrak{z}$. On the other hand, the maximality implies that the pair $(K \ltimes N, K)$ does not have non-trivial central reductions. This means that $\mathfrak{z}$ does not have $K$-invariant subspaces $\mathfrak{s}$ such that if $\tilde{\mathfrak{s}}$ denotes the orthogonal complement of $\mathfrak{s}$ on $\mathfrak{z}$ and $\tilde{N}$ is the simply connected Lie group with Lie algebra $\tilde{\mathfrak{n}}:=\tilde{\mathfrak{s}} \oplus(\mathfrak{n} / \mathfrak{z}) \simeq \mathfrak{n} / \mathfrak{s}$, then $(K \ltimes \tilde{N}, K)$ is a Gelfand pair (see [15, Section 13.4A, p. 320]).

Firstly, J. Lauret constructs a family of Gelfand pairs $(K \ltimes N, K)$ considering on $N$ a Riemannian structure (see [8]). Here $K \ltimes N$ is the group of isometries of $N$. In the particular case when $N$ is the Heisenberg group or the euclidean space $\mathbb{R}^{n}$, the commutative triples were determined in [11]. For the corresponding (matrix) spherical analysis see that article and also [2]. When additionally $N$ has square integrable representations, all the commutative triples that come from these Gelfand pairs were determined in the recent article [3].

This article has the aim of completing the classification of commutative homogeneous vector bundles associated to nilmanifolds, that is, commutative triples of the form $(K \ltimes N, K, \tau)$ with $N$ a nilpotent Lie group. We will analyze which commutative triples come from the Gelfand pairs in [13] that are not included in the list given in [8].

Acknowledgement. We are immensely grateful to Jorge Lauret, who gave impulse to this research.

\section{Preliminaries}

We recall that since we consider $N$ a two-step nilpotent Lie group, its Lie algebra splits, as a vector space, as $\mathfrak{n}=\mathfrak{z} \oplus V$, where $V$ is an orthogonal complement of the center $\mathfrak{z}$ and $[V, V] \subset \mathfrak{n}$. The group $N$ acts naturally on $\mathfrak{n}$ by the adjoint action Ad. Also, $N$ acts on $\mathfrak{n}^{*}$, the real dual space of $\mathfrak{n}$, by the dual or contragredient representation of the adjoint representation $\operatorname{Ad}^{*}(n) \lambda:=\lambda \circ \operatorname{Ad}\left(n^{-1}\right)$ for $n \in N$ and $\lambda \in \mathfrak{n}^{*}$. For each $\lambda \in \mathfrak{n}^{*}$, let $O(\lambda):=\left\{\operatorname{Ad}^{*}(n) \lambda \mid n \in N\right\}$ be its coadjoint orbit. From Kirillov's theory there is a correspondence between $\widehat{N}$ and the set of coadjoint orbits. Let $B_{\lambda}$ be the skew symmetric bilinear form on $\mathfrak{n}$ given by

$$
B_{\lambda}(X, Y):=\lambda([X, Y]) \quad \forall X, Y \in \mathfrak{n} .
$$

Let $\mathfrak{m} \subset \mathfrak{n}$ be a maximal isotropic subalgebra in the sense that $B_{\lambda}(X, Y)=0$ for all $X, Y \in \mathfrak{m}$ and let $M:=\exp (\mathfrak{m})$. Defining on $M$ the character $\chi_{\lambda}(\exp (Y)):=e^{i \lambda(Y)}$ for $Y \in \mathfrak{m}$, the irreducible representation $\rho_{\lambda} \in \widehat{N}$ associated to $O(\lambda)$ is the induced representation $\rho_{\lambda}:=\operatorname{Ind}_{M}^{N}\left(\chi_{\lambda}\right)$.

Let $X_{\lambda} \in \mathfrak{z}$ be the representative of $\left.\lambda\right|_{\mathfrak{z}}$ (the restriction of $\lambda$ to $\mathfrak{z}$ ), that is, $\lambda(Y)=\left\langle Y, X_{\lambda}\right\rangle$ for all $Y \in \mathfrak{z}$. We can split $\mathfrak{z}=\mathbb{R} X_{\lambda} \oplus \mathfrak{z} \lambda$, where $\mathfrak{z}_{\lambda}:=\operatorname{Ker}\left(\left.\lambda\right|_{\mathfrak{z}}\right)$ is the orthogonal complement of $\mathbb{R} X_{\lambda}$ in $\mathfrak{z}$.

Let $Z$ be the center of $N$. A representation $\left(\rho, H_{\rho}\right) \in \widehat{N}$ is said to be square integrable if its matrix coefficients $\langle u, \rho(x) v\rangle$, for $u, v \in H_{\rho}$, are square integrable functions on $N$ modulo $Z$.

One can see that $\rho_{\lambda} \in \widehat{N}$ is a square integrable representation if and only if $B_{\lambda}$ is non-degenerate on $V$ or, equivalently, if and only if the orbits are maximal 
(see [3]). In this situation, consider the Heisenberg algebra $\mathfrak{n}_{\lambda}:=\mathbb{R} X_{\lambda} \oplus V$ with the bracket given by

$$
[X, Y]_{\mathfrak{n}_{\lambda}}:=B_{\lambda}(X, Y) X_{\lambda} .
$$

Since the character $\chi_{\lambda}$ is trivial on $\mathfrak{z}_{\lambda}$, the representation $\rho_{\lambda}$ acts trivially on $\mathfrak{z} \lambda$ and defines an irreducible unitary representation of the corresponding Heisenberg group $N_{\lambda}$.

Now, let $P(\lambda)$ be the square root of the determinant of $\left.\left(B_{\lambda}\right)\right|_{V \times V}$, which is called the Pfaffian. This function $P$ depends only on $\left.\lambda\right|_{\mathfrak{z}}$ and so there is a homogeneous polynomial function (which we also denote $P$ ) on $\mathfrak{z}^{*}$ such that $P(\lambda)=P\left(\left.\lambda\right|_{\mathfrak{z}}\right)$ (see [15. p. 333]). According to [15, Theorem 14.2.10] there is a correspondence between the coadjoint orbits $O(\lambda)$ with $P\left(\left.\lambda\right|_{\mathfrak{z}}\right) \neq 0$ and the square integrable representations.

Apart from that, let $K$ be a compact subgroup of automorphisms of $N$ and let $K_{\lambda}$ be the stabilizer of $X_{\lambda}$ with respect to the action of $K$ on $\mathfrak{n}$. Note that since we always assume $N$ to be simply connected, we make no distinction between automorphisms of $N$ and those of $\mathfrak{n}$. It can be seen that $K_{\lambda}$ is a subgroup of the symplectic group $\operatorname{Sp}\left(V,\left.\left(B_{\lambda}\right)\right|_{V \times V}\right)$. Moreover, since $K_{\lambda}$ is compact, we can assume that it is a subgroup of the unitary group $\mathrm{U}(m) \subset \mathrm{Sp}\left(V,\left.\left(B_{\lambda}\right)\right|_{V \times V}\right)$. Let $\omega$ be the metaplectic representation of $K_{\lambda}$ associated to the Heisenberg group $N_{\lambda}$. That is,

$$
(\omega(k)(p))(z):=p\left(k^{-1} z\right)
$$

for all $p$ in the space $\mathcal{P}\left(\mathbb{C}^{m}\right)$ of polynomials on $\mathbb{C}^{m}$, where $2 m$ is the dimension on $V$. (For more details, see [3].)

For the following theorem see [3, Theorem 3] and [11, Theorem 6.1]. An important fact in the proof of this result is that the classes in $\widehat{N}$ of square integrable representations have full Plancherel measure.

Theorem 2.1. Let $N$ be a connected and simply connected real two-step nilpotent Lie group which has a square integrable representation. Let $K$ be a compact subgroup of orthogonal automorphisms of $N$ and let $\left(\tau, W_{\tau}\right) \in \widehat{K}$. Then $(K \ltimes N, K, \tau)$ is a commutative triple if and only if $\left(K_{\lambda} \ltimes N_{\lambda}, K_{\lambda},\left.\tau\right|_{K_{\lambda}}\right)$ is a commutative triple for every square integrable representation $\rho_{\lambda} \in \widehat{N}$, where $\left.\tau\right|_{K_{\lambda}}$ denotes the restriction of $\tau$ to $K_{\lambda}$. Also, $\left(K_{\lambda} \ltimes N_{\lambda}, K_{\lambda},\left.\tau\right|_{K_{\lambda}}\right)$ is a commutative triple if and only if $\omega \otimes\left(\left.\tau\right|_{K_{\lambda}}\right)$ is multiplicity free.

The previous result will be crucial, since it allows us to reduce the problem from two-step nilpotent Lie groups in general to Heisenberg groups. But it is extremely important the condition on $N$ of having square integrable representations.

There are few Gelfand pairs $(K \ltimes N, K)$ in the list of E. Vinberg such that the nilpotent group is not of the form given by J. Lauret. Specifically, they correspond to items $3,5,11,12,20$, and 26 of [13, Table 3].

In items 3 and 26 of [13, Table 3] the group $N$ does not have square integrable representations; we will omit them from our study. In the following section we will develop the analysis of the triples derived from the remaining Gelfand pairs: 
- Case A: We will develop item 5 of [13, Table 3]. Here we have $\mathfrak{n}=$ $\left[\Lambda^{2}\left(\mathbb{C}^{2 n}\right) \oplus i \mathbb{R}\right] \oplus \mathbb{C}^{2 n}$, where $\Lambda^{2}\left(\mathbb{C}^{2 n}\right)$ denotes the space of antisymmetric bilinear forms on $\mathbb{C}^{2 n}$ over the complex field, and $K=\mathrm{U}(2 n)$.

- Case B: We will study item 12 of [13, Table 3]. Denoting by $\mathbb{H}$ the quaternions, we have $\mathfrak{n}=\left[H_{0}\left(\mathbb{H}^{n}\right) \oplus \operatorname{Im}(\mathbb{H})\right] \oplus \mathbb{H}^{n}$, where $H_{0}\left(\mathbb{H}^{n}\right)$ denotes the space of hermitian $n \times n$ matrices over $\mathbb{H}$ of trace zero and $\operatorname{Im}(\mathbb{H})$ the imaginary quaternions, and $K=\mathrm{S}^{1} \times \mathrm{Sp}(n)$, where $\mathrm{S}^{1}$ is the one-dimensional torus and $\operatorname{Sp}(n)$ the symplectic group. The only difference between items 12 and 11 of [13, Table 3] is that for item 11 the group $K$ is smaller.

- Case C: We will analyze item 20 of [13, Table 3]. Here we have $\mathfrak{n}=\mathbb{R}^{7} \oplus \mathbb{R}^{8}$ and $K=\operatorname{Spin}(7)$.

\section{Analysis of COMmUtavive AND NON-COMmUtative TRIPleS}

Case A. The objects that we will describe here can be found in item 5 of 13 , Table 3] as well as in item 5 of Table 13.4.1 in the book [15].

Consider the two-step nilpotent Lie algebra

$$
\mathfrak{n}=\mathfrak{z} \oplus V=\left[\Lambda^{2}\left(\mathbb{C}^{2 n}\right) \oplus i \mathbb{R}\right] \oplus \mathbb{C}^{2 n}
$$

where the composition is given by

$$
[(z, u),(w, v)]:=\left(\left(u \wedge v, \operatorname{Im}\left(u^{*} v\right)\right), 0\right) \quad \forall z, w \in \mathfrak{z}, u, v \in V,
$$

where we denote by $u^{t}$ the row vector obtained from the column vector $u$ and by $u^{*}$ the conjugate row vector. The wedge product $u \wedge v$ can also be expressed as $u v^{t}-v u^{t}$.

The unitary group $K=\mathrm{U}(2 n)$ acts on $\mathfrak{z}$ by $k \cdot\left(u v^{t}-v u^{t}, s\right):=\left(k\left(u v^{t}-v u^{t}\right) k^{t}, s\right), \quad \forall k \in K$ and $\forall u v^{t}-v u^{t} \in \Lambda^{2}\left(\mathbb{C}^{2 n}\right), s \in i \mathbb{R}$.

We consider in particular a linear functional $\lambda$ in $\mathfrak{n}^{*}$ such that (when we restrict it to $\mathfrak{z})$ it has as a representative the element $X_{\lambda}:=(u \wedge v, 0) \in \mathfrak{z}$ with $u \wedge v$ non-degenerate. Let $\rho_{\lambda}$ denote the irreducible representation class associated to $\lambda$. From the formula for the Pfaffian given in [15, pp. 339-340 ], $P(\lambda)$ is a positive multiple of the determinant of the matrix $u v^{t}-v u^{t}$. According to our election of the linear functional $\lambda, P(\lambda)$ results invertible. Therefore $\rho_{\lambda}$ is square integrable and we are allowed to apply Theorem 2.1. In this case, the subgroup $K_{\lambda}$ coincides with the symplectic group $\operatorname{Sp}(n)$.

Let $\tau \in \widehat{K}$ be non-trivial. Then there is a non-trivial $\eta \in \widehat{\operatorname{Sp}(n)}$ appearing in the restriction of $\tau$ to $K_{\lambda}$.

Now we introduce some notation. Let $\delta_{i, j}$ denote the Kronecker delta. Writing $H_{i}:=\delta_{i, i}-\delta_{n+i, n+i}$, we have that the Cartan subalgebra $\mathfrak{h}$ of $\mathfrak{s p}(n)$ is a complex vector space generated by $\left\{H_{1}, \ldots, H_{n}\right\}$. Let $\left\{L_{1}, \ldots, L_{n}\right\}$ be its dual basis in the dual space $\mathfrak{h}^{*}$, so $\left\langle L_{i}, H\right\rangle=h_{i}$ for all $H \in \mathfrak{h}$. From the theorem of the highest weight, every irreducible representation of $\mathfrak{s p}(n)$ is in correspondence with a nonnegative integer linear combination of the fundamental weights. Hence $\eta \in \widehat{\operatorname{Sp}(n)}$ can be parametrized in terms of the weights $\left\{L_{i}\right\}$ as $\left(\eta_{1}, \ldots, \eta_{n}\right)$, where $\eta_{i} \in \mathbb{Z}_{\geq 0} \forall i$ and $\eta_{1} \geq \eta_{2} \geq \ldots \geq \eta_{n}$. (For a reference see, for example, [4, 6].) We will denote 
the representation $\eta \in \widehat{\operatorname{Sp}(n)}$ by $\eta_{\left(\eta_{1}, \ldots, \eta_{n}\right)}$ to emphasize that the representation $\eta$ is in correspondence with the tuple $\left(\eta_{1}, \ldots, \eta_{n}\right)$ that we call partition.

The metaplectic representation $\left(\omega, \mathcal{P}\left(\mathbb{C}^{2 n}\right)\right)$ of $\operatorname{Sp}(n)$ decomposes (using the notation given in the previous sections) as

$$
\omega=\bigoplus_{j \in \mathbb{Z}_{\geq 0}} \eta_{(j)}
$$

where each $\eta_{(j)}$ corresponds to the partition of length one $(j)$, for some non-negative integer $j$.

The following fact is proved in [3, Corollary 2] and will be useful to deduce our main results.

Lemma 3.1. Let $\eta$ be an irreducible representation of $\operatorname{Sp}(n)$. Then $\eta$ appears in the decomposition into irreducible factors of $\eta \otimes \eta_{(2)}$.

From the above lemma, the representation $\eta$ appears in the decomposition of the factors $\eta \otimes \eta_{(0)}$ and $\eta \otimes \eta_{(2)}$. Then, it appears with multiplicity in the decomposition into irreducible factors of $\left.\omega \otimes \tau\right|_{K_{\lambda}}$. Therefore, by Theorem 2.1. we have the following result.

Theorem 3.2. The triple $(K \ltimes N, K, \tau)$, where $N$ is the simply connected nilpotent Lie group with Lie algebra $\left[\Lambda^{2}\left(\mathbb{C}^{2 n}\right) \oplus i \mathbb{R}\right] \oplus \mathbb{C}^{2 n}$ and $K=\mathrm{U}(2 n)$, is commutative if and only if $\tau$ is the trivial representation.

Case B. The objects that we will describe here can be found in item 12 of [13, Table 3] as well as in item 9 of Table 13.4.1 in the book [15].

Consider the two-step nilpotent Lie algebra

$$
\mathfrak{n}=\mathfrak{z} \oplus V:=\left[H_{0}\left(\mathbb{H}^{n}\right) \oplus \operatorname{Im}(\mathbb{H})\right] \oplus \mathbb{H}^{n}
$$

with Lie bracket given by

$$
[(z, u),(w, v)]:=\left(\left(\left(u i v^{*}-v i u^{*}\right)_{0}, u^{*} v-v^{*} u\right), 0\right)
$$

for all $z, w \in \mathfrak{z}=H_{0}\left(\mathbb{H}^{n}\right) \oplus \operatorname{Im}(\mathbb{H})$ and $u, v \in V=\mathbb{H}^{n}$, where, in general, if $A$ is a matrix, $(A)_{0}:=A-\frac{1}{2} \operatorname{tr}(A) I$. Let $N$ be the simply connected nilpotent Lie group with Lie algebra $\mathfrak{n}$.

Every point $\left(e^{i \theta}, k\right) \in K=\mathrm{S}^{1} \times \mathrm{Sp}(n)$ acts on $\mathfrak{n}$ by

$$
\left(e^{i \theta}, k\right) \cdot((A, q), v):=\left(\left(k A k^{*}, e^{i \theta} q e^{-i \theta}\right), k v e^{-i \theta}\right),
$$

for all $A \in H_{0}\left(\mathbb{H}^{n}\right), q \in \operatorname{Im}(\mathbb{H})$, and $v \in \mathbb{H}^{n}$.

Let $\tau=\eta \otimes \chi_{r}$, where $\eta \in \widehat{\operatorname{Sp}(n)}$ and $\chi_{r}$ is a character of $\mathrm{S}^{1}$.

First we consider the case where the factor in $\widehat{\operatorname{Sp}(n)}$ is trivial, that is, $\tau=\chi_{r}$. We recall that the convolution algebra $L_{\tau, \tau}^{1}\left(K \ltimes N, \operatorname{End}\left(W_{\tau}\right)\right)$ is naturally identified with the space of $\operatorname{End}\left(W_{\tau}\right)$-valued integrable functions on $N$ such that $F(k \cdot x)=$ $\tau(k) F(x) \tau(k)^{-1}$ for all $k \in K$ and $x \in N$ (see e.g. [2, 11]). In this situation, it totally coincides with the convolution algebra of $K$-invariant integrable scalar functions on $N$, which is commutative since we have a Gelfand pair. Therefore we have commutative triples. 
Now let $\eta \in \widehat{\operatorname{Sp}(n)}$ be non-trivial. We consider the linear functional $\lambda$ with representative $X_{\lambda}:=(0, q) \in \mathfrak{z}$. Let $\rho_{\lambda}$ denote the irreducible representation class associated to $\lambda$. From the formula for the Pfaffian given in [15, p. 340], $P(\lambda)$ is a positive multiple of $|q|^{2 n}$. Then for all imaginary non-zero quaternions $q$, the associated representation $\rho_{\lambda}$ is square integrable. The group $K_{\lambda}$ is easily calculated: If the quaternion $q$ belongs to $i \mathbb{R}, K_{\lambda}$ coincides with $K$ (since $q$ commutes with every complex); for the other cases, $K_{\lambda}$ is $\operatorname{Sp}(n)$. We consider for example $q=j \in \operatorname{Im}(\mathbb{H})$ in order to fix $K_{\lambda}=\operatorname{Sp}(n)$. Note that $\left.\tau\right|_{K_{\lambda}}=\eta$.

The metaplectic representation $\left(\omega, \mathcal{P}\left(\mathbb{C}^{2 n}\right)\right)$ of $K_{\lambda}=\operatorname{Sp}(n)$ decomposes as

$$
\omega=\bigoplus_{j \in \mathbb{Z}_{\geq 0}} \eta_{(j)} .
$$

Therefore $\omega \otimes \eta$ is not multiplicity free: $\eta$ appears in the factors $\eta \otimes \eta_{(0)}$ and in $\eta \otimes \eta_{(2)}$ by Lemma 3.1. Then, by Theorem 2.1, in these cases the triples are not commutative.

Theorem 3.3. The triple $(K \ltimes N, K, \tau)$, where $N$ is the simply connected nilpotent Lie group with Lie algebra $\left[H_{0}\left(\mathbb{H}^{n}\right) \oplus \operatorname{Im}(\mathbb{H})\right] \oplus \mathbb{H}^{n}$ and $K=\mathrm{S}^{1} \times \mathrm{Sp}(n)$, is commutative if and only if $\tau \in \widehat{\mathrm{S}^{1}}$.

To conclude this case we want to note that the analysis of item 11 of [13, Table 3] is the same (or simpler), since the subgroup $K$ is only $\operatorname{Sp}(n)$.

Case C. The objects that we will describe here can be found in item 20 of [13, Table 3], as well as in item 2 of Table 13.4.1 in the book [15.

In this case we study an H-type group ([5]). Consider the two-step nilpotent Lie algebra $\mathfrak{n}=\mathfrak{z} \oplus V$, where $V$ is $\mathbb{R}^{8}$ or the octonions $\mathbb{O}$ and the center $\mathfrak{z}$ is $\mathbb{R}^{7}$ or the imaginary octonions $\operatorname{Im}(\mathbb{O})$ with Lie bracket on $V$ characterized by $\langle[u, v], z\rangle_{\mathfrak{z}}:=\langle J(z) u, v\rangle_{V}$, where $(J, V)$ is a real representation of $\mathfrak{z}$ given by the product of octonions $J(z) v:=z v$ for $z \in \operatorname{Im}(\mathbb{O})$ and $v \in \mathbb{O}$.

Let $K$ be the maximal connected group of orthogonal automorphisms of $N$. Precisely, $K=\operatorname{Spin}(7)$. (We mention that in general, for an H-type group, its group of automorphisms was determined by L. Saal in [12.)

Let $\lambda$ be a linear functional with representative $X \in \mathfrak{z}$. From [15, p. 340], the Pfaffian $P(\lambda)$ is not null almost everywhere. In this case, $K_{\lambda}$ is isomorphic to the spin group $\operatorname{Spin}(6)$. Therefore we will work with the Lie algebra $\mathfrak{s o}(6)$.

Let $\tau$ be a non-trivial irreducible unitary representation of $\operatorname{Spin}(7)$. When we restrict $\tau$ to $\operatorname{Spin}(6)$ it decomposes as a sum of irreducible representations and we can pick one non-trivial factor, which we will denote as $\eta \in \operatorname{Spin}(6)$. We can identify $\eta$ with its derived representation or we can as well view it as a representation of $\mathfrak{s o}(6)$.

Apart from that, it is easy to derive the decomposition of the metaplectic representation, since $\operatorname{Spin}(6)$ is isomorphic to $\mathrm{SU}(4)$ and it is well known that its action on $\mathcal{P}\left(\mathbb{C}^{4}\right)$ decomposes into $\bigoplus_{j \in \mathbb{Z}_{\geq 0}} \mathcal{P}_{j}\left(\mathbb{C}^{4}\right)$. The metaplectic representation can be 
identified with

$$
\omega=\bigoplus_{j \in \mathbb{Z}_{\geq 0}} \tilde{\eta}_{(j)}
$$

where $\tilde{\eta}_{(j)}$ corresponds to the partition of length one $(j)$ of $\mathfrak{s o}(6)$. Here we decided to use a notation analogous to that of the representations of $\mathfrak{s p}(n)$. As a consequence of the highest weight theorem, we can associate each irreducible representation $\sigma$ of $\mathfrak{s o}(2 m)$ with an $n$-tuple of integers $\left(\sigma_{1}, \ldots, \sigma_{m}\right)$ satisfying the condition $\sigma_{1} \geq \sigma_{2} \geq$ $\ldots \geq \sigma_{m-1} \geq\left|\sigma_{m}\right|$ (see [4, 10]). The following fact can be found in [10, Theorem 3.2 and Remark 3.5].

Lemma 3.4. Let $\tilde{\eta}$ be an arbitrary irreducible representation of $\mathfrak{s o}(2 m)$ associated to a sequence of $m$ integers $\tilde{\eta}_{1} \geq \tilde{\eta}_{2} \geq \ldots \geq \tilde{\eta}_{m-1} \geq\left|\tilde{\eta}_{m}\right|$ and let $s$ be a non-negative integer. Then the multiplicity of an irreducible representation $\sigma$ (associated to the sequence of integers $\left.\sigma_{1} \geq \sigma_{2} \geq \ldots \geq \sigma_{m-1} \geq\left|\sigma_{m}\right|\right)$ in the tensor product $\tilde{\eta} \otimes \tilde{\eta}_{(s)}$ is equal to the number of integer sequences $\varsigma$ satisfying:

(i) $\varsigma_{1} \geq \varsigma_{2} \geq \ldots \geq \varsigma_{m-1} \geq\left|\varsigma_{m}\right|$;

(ii) $\tilde{\eta}_{1} \geq \varsigma_{1} \geq \tilde{\eta}_{2} \geq \varsigma_{2} \geq \ldots \geq \varsigma_{m-1} \geq \tilde{\eta}_{m} \geq \varsigma_{m}$ and $\sigma_{1} \geq \varsigma_{1} \geq \sigma_{2} \geq \varsigma_{2} \geq \ldots \geq$ $\varsigma_{m-1} \geq \sigma_{m} \geq \varsigma_{m}$

(iii) $\sum_{i=1}^{m}\left(\tilde{\eta}_{i}-\varsigma_{i}\right)+\sum_{i=1}^{m}\left(\sigma_{i}-\varsigma_{i}\right)=s$;

(iv) $\varsigma_{m} \in\left\{\tilde{\eta}_{m}, \sigma_{m}\right\}$.

Now we will apply this lemma with $m=3$ to a fixed non-trivial irreducible representation $\tilde{\eta}$ in the decomposition of $\left.\tau\right|_{K_{\lambda}}$, to deduce whether the tensor product $\left.\omega \otimes \tau\right|_{K_{\lambda}}$ is multiplicity free or not. The representation $\tilde{\eta}$ corresponds to $\left(\tilde{\eta}_{1}, \tilde{\eta}_{2}, \tilde{\eta}_{3}\right)$ with $\tilde{\eta}_{i} \in \mathbb{Z}$ for $i=1,2,3, \tilde{\eta}_{1} \geq \tilde{\eta}_{2} \geq\left|\tilde{\eta}_{3}\right|$, and $\tilde{\eta}_{1}>0$ since it is non-trivial.

- If $\tilde{\eta}_{1}>\tilde{\eta}_{3}$ (in particular if $\left.\tilde{\eta}_{3} \leq 0\right)$, then $\tilde{\eta}$ appears in $\tilde{\eta} \otimes \tilde{\eta}_{s}$ for $s=2\left(\tilde{\eta}_{1}-\right.$ $\left.\tilde{\eta}_{3}\right)>0$, since $\varsigma=\left(\tilde{\eta}_{2}, \tilde{\eta}_{3}, \tilde{\eta}_{3}\right)$ satisfies the conditions listed in Lemma 3.4 By (3.1) we have that $\tilde{\eta}$ appears at least twice in $\left.\omega \otimes \tau\right|_{K_{\lambda}}$.

- If $\tilde{\eta}_{1}=\tilde{\eta}_{2}=\tilde{\eta}_{3}=r$ for $r \in \mathbb{Z}_{>0}$, then if $\sigma=\left(\sigma_{1}, \sigma_{2}, \sigma_{3}\right)$ appears in $\tilde{\eta} \otimes \tilde{\eta}_{s}$ for $s \in \mathbb{Z}_{\geq 0}$, then by Lemma $3.4 \sigma_{1}=\sigma_{2}=r$. Also, with the notation in that Lemma, it must happen that $\varsigma$ is $(r, r, r)$ or $\left(r, r, \sigma_{3}\right)$. For the first case, $\sigma_{3}-r=s$; then, since $r \geq \sigma_{3}$, the only possibility for $s$ is $s=0$. For the second case, $r-\sigma_{3}=s$. Therefore $s$ is completely determined by $\sigma$. Thus $\sigma=\left(r, r, \sigma_{3}\right)$ with $r \geq \sigma_{3}$ appears once in $\tilde{\eta} \otimes \oplus_{j \in \mathbb{Z}_{>0}} \tilde{\eta}_{(j)}$. Consequently, if $\tilde{\eta}=(r, r, r)$ and $\sigma=\left(r, r, \sigma_{3}\right)$ with $r>\sigma_{3}$ both appear in $\left.\tau\right|_{K_{\lambda}}$, the tensor product $\left.\omega \otimes \tau\right|_{K_{\lambda}}$ is not multiplicity free. Note that in this situation $\sigma$ satisfies the conditions $\tilde{\eta}$ for the first item. Therefore we have the following conclusion.

Theorem 3.5. The triple $(K \ltimes N, K, \tau)$, where $N$ is the simply connected nilpotent Lie group with Lie algebra $\operatorname{Im}(\mathbb{O}) \oplus \mathbb{O}$ and $K=\operatorname{Spin}(7)$, is commutative if and only if $\left.\tau\right|_{\operatorname{Spin}(6)}$ is associated to a partition of the form $(r, r, r)$ for $r \in \mathbb{Z}_{\geq 0}$.

\section{Conclusions}

We can sum up as follows. Theorem 3.2 does not provide non-trivial commutative triples. Theorem 3.3 gives rise to a commutative triple only if $\tau$ is a character 
of $\mathrm{S}^{1}$. These cases are twisted Gelfand pairs. Finally, Theorem 3.5 gives a family of commutative triples. This family is similar to the case of the H-type group listed by J. Lauret in $\left[8\right.$. There the nilpotent Lie algebra $\mathfrak{n}$ is $\operatorname{Im}(\mathbb{H}) \oplus \mathbb{H}^{n}$, where $\operatorname{Im}(\mathbb{H})$ is its center $\mathfrak{z}$ and the Lie bracket on $\mathbb{H}^{n}$ is given, analogously to case $\mathrm{C}$, by the real representation of $\mathfrak{z}, J(z)(v):=\left(z v_{1}, \ldots, z v_{n}\right)$ for $v=\left(v_{1}, \ldots, v_{n}\right) \in \mathbb{H}$. Its (maximal connected) group of automorphisms is $K=\mathrm{SU}(2) \times \mathrm{Sp}(n)$. By [3 Proposition 1], in that case we obtain commutative triples if and only if $\tau \in \widehat{\operatorname{SU}(2)}$ or $\tau \in \widehat{\operatorname{Sp}(n)}$ corresponding to a partition of the form $(a, a, \ldots, a)$ of length at most $n$ for a non-negative integer $a$.

Let $\mathrm{H}_{n}$ be the Heisenberg group $\mathbb{C}_{n} \times \mathbb{R}$, and let $N$ be a simply connected nilpotent Lie group having square integrable representations. In conclusion, if we exclude from the analysis the Gelfand pairs of the form $\left(\mathrm{H}_{n} \ltimes K, K\right)$, where $K$ is a proper subgroup of $\mathrm{U}(n)$, all the commutative triples of the form $(K \ltimes N, K, \tau)$, where $K$ is a compact subgroup of $G$, are the following:

(1) $\left(\left[\mathrm{S}^{1} \times \mathrm{Sp}(n)\right] \ltimes N, \mathrm{~S}^{1} \times \mathrm{Sp}(n), \tau\right)$, where $N$ is the simply connected nilpotent Lie group with Lie algebra $\left[H_{0}\left(\mathbb{H}^{n}\right) \oplus \operatorname{Im}(\mathbb{H})\right] \oplus \mathbb{H}^{n}$, is commutative for $\tau \in \widehat{\mathrm{S}^{1}}$. It is a twisted Gelfand pair. It corresponds to the preceding case $\mathrm{C}$.

(2) $\left(\mathrm{SU}(n) \times \mathbb{S}^{1}, N\left(\mathfrak{s u}(n), \mathbb{C}^{n}\right), \tau\right)$, for all $\tau \in \widehat{\mathbb{S}^{1}}$, where $n \geq 3$. It is a twisted Gelfand pair.

(3) $\left(\mathrm{SU}(n) \times \mathbb{S}^{1}, N\left(\mathfrak{u}(n), \mathbb{C}^{n}\right), \tau\right)$, for all $\tau \in \widehat{\mathbb{S}^{1}}$, where $n \geq 3$. It is a twisted Gelfand pair.

(4) $(\operatorname{Spin}(7) \ltimes N, \operatorname{Spin}(7), \tau)$, where $N$ is the simply connected nilpotent Lie group with Lie algebra $\operatorname{Im}(\mathbb{O}) \oplus \mathbb{O}$, is commutative when $\left.\tau\right|_{\operatorname{Spin}(6)}$ is associated to a constant partition $(r, r, r)$ for $r \in \mathbb{Z}_{\geq 0} . N$ is of Heisenberg type. It corresponds to the preceding case $\mathrm{C}$.

(5) $\left(\mathrm{SU}(2) \times \mathrm{Sp}(n), N\left(\mathfrak{s u}(2),\left(\mathbb{C}^{2}\right)^{n}\right), \tau\right)$, for all $\tau \in \widehat{\mathrm{SU}(2)}$ and for all $\tau \in \widehat{\operatorname{Sp}(n)}$ associated to a constant partition of length at most $n$, where $n \geq 1$. $N\left(\mathfrak{s u}(2),\left(\mathbb{C}^{2}\right)^{n}\right)$ is of Heisenberg type.

(6) $\left(\mathrm{SU}(2) \times \mathrm{U}(k) \times \mathrm{Sp}(n), N\left(\mathfrak{u}(n),\left(\mathbb{C}^{2}\right)^{k} \oplus\left(\mathbb{C}^{2}\right)^{n}\right), \tau\right)$, for all $\tau \in \widehat{\mathrm{U}(k)}$, where $k \geq 1, n \geq 0$.

(7) $(G \times U, N(\mathfrak{g}, V), \tau)$, where $\mathfrak{g}=\mathfrak{s u}\left(m_{1}\right) \oplus \ldots \oplus \mathfrak{s u}\left(m_{\beta}\right) \oplus \mathfrak{s u}(2) \oplus \ldots \oplus \mathfrak{s u}(2) \oplus \mathfrak{c}$ with $\mathfrak{c}$ an abelian component, $G=\mathrm{SU}\left(m_{1}\right) \times \ldots \times \mathrm{SU}\left(m_{\beta}\right) \times \mathrm{SU}(2) \times$ $\ldots \times \mathrm{SU}(2), V=\mathbb{C}^{m_{1}} \oplus \ldots \oplus \mathbb{C}^{m_{\beta}} \oplus \mathbb{C}^{2 k_{1}+2 n_{1}} \oplus \ldots \oplus \mathbb{C}^{2 k_{\alpha}+2 n_{\alpha}}$, and $U=\mathbb{S}^{1} \times \ldots \times \mathbb{S}^{1} \times \mathrm{U}\left(k_{1}\right) \times \mathrm{Sp}\left(n_{1}\right) \times \ldots \times \mathrm{U}\left(k_{\alpha}\right) \times \operatorname{Sp}\left(n_{\alpha}\right)$, for all $\tau \in$ $\widehat{\mathbb{S}^{1}} \otimes \ldots \otimes \widehat{\mathbb{S}^{1}} \otimes \widehat{\mathrm{U}\left(k_{1}\right)} \otimes \ldots \otimes \widehat{\mathrm{U}\left(k_{\alpha}\right)}$, where $m_{j} \geq 3$ for all $1 \leq j \leq \beta$, and $k_{i} \geq 1, n_{i} \geq 0$ for all $1 \leq i \leq \alpha$.

(8) $\left(\mathrm{H}_{n} \ltimes \mathrm{U}(n), \mathrm{U}(n), \tau\right)$ for all $\tau \in \widehat{\mathrm{U}(n)}$ (proved by Yakimova in [17]). This is the unique strong Gelfand pair of this form.

In items $2,3,5,6$, and 7 above, the nilpotent Lie group $N=N(\mathfrak{g}, V)$ is endowed with a left-invariant Riemannian metric determined by an inner product $\langle\cdot, \cdot\rangle$ on its Lie algebra $\mathfrak{n}$ described as follows. Let $(\pi, V)$ be a faithful real representation 
of a compact Lie algebra $\mathfrak{g}$. We consider inner products $\langle\cdot, \cdot\rangle_{\mathfrak{g}}$ on $\mathfrak{g}$ and $\langle\cdot, \cdot\rangle_{V}$ on $V$ such that $\langle\cdot, \cdot\rangle_{\mathfrak{g}}$ is $\operatorname{ad}(\mathfrak{g})$-invariant and $\langle\cdot, \cdot\rangle_{V}$ is $\pi(\mathfrak{g})$-invariant. Let $\mathfrak{n}:=\mathfrak{g} \oplus V$ be the two-step nilpotent Lie algebra with center $\mathfrak{g}$ and Lie bracket defined on $V$ by $\langle[u, v], X\rangle_{\mathfrak{g}}:=\langle\pi(X) u, v\rangle_{V}$ for all $u, v \in V, X \in \mathfrak{g}$. These inner products define an inner product $\langle\cdot, \cdot\rangle$ on $\mathfrak{n}$ satisfying $\left.\langle\cdot, \cdot\rangle\right|_{\mathfrak{g} \times \mathfrak{g}}=\langle\cdot, \cdot\rangle_{\mathfrak{g}},\left.\langle\cdot, \cdot\rangle\right|_{V \times V}=\langle\cdot, \cdot\rangle_{V}$, and $\langle\mathfrak{g}, V\rangle=0$. Let $N(\mathfrak{g}, V)$ be the connected, simply connected, two-step nilpotent Lie group with Lie algebra $\mathfrak{n}$. Specifically, in item $2, \mathbb{C}^{n}$ denotes the standard representation of $\mathfrak{s u}(n)$; in item $3, \mathbb{C}^{n}$ denotes the standard representation of $\mathfrak{u}(n)$ regarded as a real representation; in item $5, \mathbb{C}^{2}$ denotes the standard representation of $\mathfrak{s u}(2)$ regarded as a real representation; in item $6,\left(\mathbb{C}^{2}\right)^{k} \oplus\left(\mathbb{C}^{2}\right)^{n}$ is an orthogonal sum, the center of $\mathfrak{u}(2)$ acts non-trivially only on $\left(\mathbb{C}^{2}\right)^{k},\left(\mathbb{C}^{2}\right)^{n}$ denotes the representation of $\mathfrak{s u}(2)$ stated in the first item, $\mathfrak{u}(2)$ acts component-wise on $\left(\mathbb{C}^{2}\right)^{k}$ in the standard way regarded as a real representation, and $\tau \in \widehat{\mathrm{U}(k)}$; and in item 7 , $\mathfrak{g}$ is acting on $V$ as follows: For each $1 \leq i \leq \beta+\alpha, \mathfrak{c}$ has a unique subspace $\mathfrak{c}_{i}$ acting non-trivially on only the $i$-th component of $V$ and the dimension of $\mathfrak{c}_{i}$ is 1 . For $1 \leq i \leq \beta, \mathfrak{s u}\left(m_{i}\right) \oplus \mathfrak{c}_{i}$ (which is isomorphic to $\mathfrak{u}\left(m_{i}\right)$ ) acts non-trivially only on $\mathbb{C}^{m_{i}}$. For $\beta+1<i \leq \beta+\alpha, \mathfrak{s u}(2) \oplus \mathfrak{c}_{i}$ acts non-trivially only on $\mathbb{C}^{2 k_{i}+2 n_{i}}$ as in the above case. For more details, see [3, [8, 9].

\section{REFERENCES}

[1] C. Benson, J. Jenkins and G. Ratcliff, On Gel'fand pairs associated with solvable Lie groups, Trans. Amer. Math. Soc. 321 (1990), no. 1, 85-116. MR 1000329

[2] R. Díaz Martín and F. Levstein, Spherical analysis on homogeneous vector bundles of the 3-dimensional euclidean motion group, Monatsh. Math. 185 (2018), no. 4, 621-649. MR 3777423

[3] R. Díaz Martín and L. Saal, Matrix spherical analysis on nilmanifolds, Transform. Groups 24 (2019), no. 3, 887-911. MR 3989695

[4] W. Fulton and J. Harris, Representation theory. A first course, Graduate Texts in Mathematics, 129, Springer-Verlag, New York, 1991. MR 1153249

[5] A. Kaplan, Fundamental solutions for a class of hypoelliptic PDE generated by composition of quadratic forms, Trans. Amer. Math. Soc. 258 (1980), no. 1, 147-153. MR 0554324

[6] A. W. Knapp, Lie groups beyond an introduction, second edition, Progress in Mathematics, 140, Birkhäuser Boston, Boston, MA, 2002. MR 1920389

[7] A. Korányi, Some applications of $\mathrm{Gel}^{\prime}$ fand pairs in classical analysis, in Harmonic analysis and group representations, 333-348, Liguori, Naples, 1982. MR 0777343

[8] J. Lauret, Gelfand pairs attached to representations of compact Lie groups, Transform. Groups 5 (2000), no. 4, 307-324. MR 1800530

[9] J. Lauret, Homogeneous nilmanifolds attached to representations of compact Lie groups, Manuscripta Math. 99 (1999), no. 3, 287-309. MR 1702605.

[10] S. Okada, Pieri rules for classical groups and equinumeration between generalized oscillating tableaux and semistandard tableaux, Electron. J. Combin. 23 (2016), no. 4, Paper 4.43, 27 pp. MR 3604801

[11] F. Ricci and A. Samanta, Spherical analysis on homogeneous vector bundles, Adv. Math. 338 (2018), 953-990. MR 3861720 
[12] L. Saal, The automorphism group of a Lie algebra of Heisenberg type, Rend. Sem. Mat. Univ. Politec. Torino 54 (1996), no. 2, 101-113. MR 1490017

[13] È. B. Vinberg, Commutative homogeneous spaces and co-isotropic symplectic actions, Russian Math. Surveys 56 (2001), no. 1, 1-60; translated from Uspekhi Mat. Nauk 56 (2001), no. 1(337), 3-62. MR 1845642

[14] N. R. Wallach, Harmonic analysis on homogeneous spaces, Marcel Dekker, New York, 1973. MR 0498996

[15] J. A. Wolf, Harmonic analysis on commutative spaces, Mathematical Surveys and Monographs, 142, American Mathematical Society, Providence, RI, 2007. MR 2328043

[16] J. A. Wolf, On the analytic structure of commutative nilmanifolds, J. Geom. Anal. 26 (2016), no. 2, 1011-1022. MR 3472827

[17] O. Yakimova, Principal Gelfand pairs, Transform. Groups 11 (2006), no. 2, 305-335. MR 2231190

Rocio Díaz Martín ${ }^{\bowtie}$

CONICET and Universidad Nacional de Córdoba

rocio.diazmartin@unc.edu.ar

Linda Saal

Universidad Nacional de Córdoba

saal@mate. uncor. edu

Received: August 21, 2019

Accepted: February 17, 2020 